\title{
A survey on exponential random graph models: an application perspective
}

\author{
Saeid Ghafouri ${ }^{1}$, Seyed Hossein Khasteh ${ }^{\text {Corresp. } 1}$ \\ ${ }^{1}$ School of computer engineering, K. N. Toosi University of Technology, Tehran, Iran \\ Corresponding Author: Seyed Hossein Khasteh \\ Email address: khasteh@kntu.ac.ir
}

The uncertainty underlying real-world phenomena has attracted attention toward statistical analysis approaches. In this regard, many problems can be modeled as networks. Thus, the statistical analysis of networked problems has received special attention from many researchers in recent years. Exponential Random Graph Models known as ERGMs are one of the popular statistical methods for analyzing the graphs of networked data. ERGM is a generative statistical network model whose ultimate goal is to present a subset of networks with particular characteristics as a statistical distribution. In the context of ERGMs, these graph's characteristics are called statistics or configurations. Most of the time, they are the number of repeated subgraphs across the graphs. Some examples include the number of triangles or the number of cycle of an arbitrary length. Also, any other census of the graph, as with the edge density, can be considered as one of the graph's statistics. In this review paper, after explaining the building blocks and classic methods of ERGMs, we have reviewed their newly presented approaches and research papers. Further, we have conducted a comprehensive study on the applications of ERGMs in many research areas which to the best of our knowledge has not been done before. This review paper can be used as an introduction for scientists from various disciplines whose aim is to use ERGMs in some networked data in their field of expertise. 
1 A Survey on Exponential Random Graph Models: An

2 Application Perspective

3

4

5

6

7

8

9

10

11

12

13

14

15

16

17

18

19

20

21

22

23

24

25

26

27

28

29

30

31

32

33

34

35

36

37

38

39

Saeid Ghafouri ${ }^{1}$, Seyed Hossein Khasteh ${ }^{2}$

${ }^{1}$ School of computer engineering, K. N. Toosi university of technology, Tehran, Iran

${ }^{2}$ School of computer engineering, K. N. Toosi university of technology, Tehran, Iran

Corresponding Author:

Seyed Hossein Khasteh ${ }^{1}$

Faculty of computer engineering, K. N. Toosi university of technology, Seyed Khandan Bridge, Tehran, Iran

Email address: khasteh@kntu.ac.ir

\section{Abstract}

The uncertainty underlying real-world phenomena has attracted attention toward statistical analysis approaches. In this regard, many problems can be modeled as networks. Thus, the statistical analysis of networked problems has received special attention from many researchers in recent years. Exponential Random Graph Models known as ERGMs are one of the popular statistical methods for analyzing the graphs of networked data. ERGM is a generative statistical network model whose ultimate goal is to present a subset of networks with particular characteristics as a statistical distribution. In the context of ERGMs, these graph's characteristics are called statistics or configurations. Most of the time, they are the number of repeated subgraphs across the graphs. Some examples include the number of triangles or the number of cycle of an arbitrary length. Also, any other census of the graph, as with the edge density, can be considered as one of the graph's statistics. In this review paper, after explaining the building blocks and classic methods of ERGMs, we have reviewed their newly presented approaches and research papers. Further, we have conducted a comprehensive study on the applications of ERGMs in many research areas which to the best of our knowledge has not been done before. This review paper can be used as an introduction for scientists from various disciplines whose aim is to use ERGMs in some networked data in their field of expertise.

\section{Introduction}

Networks are an essential part of everyday life. From the World Wide Web to biological networks, they all shape the connections of the world. There are many examples of the use of networks in various fields and disciplines. Examples of them include social networks, traffic systems, and disease spread networks. The most canonical way of representing a network is a graph. Indeed,

PeerJ Comput. Sci. reviewing PDF | (CS-2019:04:37112:1:2:NEW 5 Mar 2020) 
40 not all of the networks' ties are presented with $100 \%$ certainty. For example, in a friendship 41 network, the level of friendship is not the same among all individuals or there is always a chance 42 that two friends stop their friendship in the future Further, in some domains, the current snapshots 43 of the network depend on its timestamp where the network's shape might be different if the 44 snapshot has been taken in another time. For example, in a blockchain network, the structure of 45 the network connections is constantly changing. Hence, the graph has a dynamic structure over time. All of these suggest some level of uncertainty in many real-world networks. Therefore, simple graph theory will not suffice for examining these networks. These limitations have led to proposing completely new statistical approaches for graph analysis. More specifically, we want to build a statistical model based on the observed dataset. In these types of graph analysis, a probability in the interval of [0,1] is assigned to each graph. If this probability is close to zero, it indicates that the graph has no chance of existence, while one suggests that this particular graph will undoubtedly exist in the generated data. Any other value between zero and one indicates the existence probability of that graph. These probabilities have different meanings depending on the domain of the network. However, the probability of graph's existence is the most fundamental definition, to which we will stick for the rest of the article. Statistical graphs (Frank, 1981; Robins et al., 2007a; Goldenberg et al., 2010) have attracted scientists from different disciplines. There are different kinds of approaches regarding their formulation and learning methods. People from mathematics, computer science, physics, and of course statistics have proposed different algorithms and methods for designing the framework for statistically modeled graphs. In addition, statistical graphs are also fundamental to generative models for generating new graphs with similar statistics and attributes to the original graphs. These artificial generated models have various applications, e.g., data augmentation for learning systems where we have datasets with limited resources or simulating and predicting other possible graphs with similar properties. Furthermore, there are longitudinal (Holland \& Leinhardt, 1977; Koskinen \& Snijders, 2007; de la Haye et al., 2017; Block et al., 2018) models which aim to observe a network over a time period and predict the network's future dynamics. Although different approaches exist, in this work, we are going to review research articles about a particular family of statistical graphs known as Exponential Random Graph Models, abbreviated as ERGM. Designing a statistical model consists of three steps: (1) Designing a general formulation based on the context and statistical specification of the dataset; (2) Estimating the parameters of the designed model via some learning methods, where sometimes this step is addressed as the phase of fitting the model to the data; and (3) employing the model with learned parameters to predict the future or unseen part of the data, generation of new data with similar properties, or any other possible tasks. The model utilized for ERGMs (step 1) is almost similar across the entire literature. However, the parameter estimation step (step 2) differs case by case. Figure 1 (in Supplemental Files) demonstrates the mentioned steps' flowchart. formation between two nodes. In ERGMs, more complex structures with a reasonable level of dependence have also been taken into account. This approach has led to more complicated models 
80

81

82

83

84

85

86

87

88

89

90

91

92

93

94

95

96

97

98

99

100

101

102

103

104

105

106

107

108

109

110

111

112

113

114

115

116

117

118

119

which also require more sophisticated learning methods. Additionally, due to the better accuracy of the models with dependent structures, they are applicable to a more considerable extent of the problems. Therefore, there is a rising interest in using ERGMs in multiple research areas. Previous surveys (Anderson, Wasserman \& Crouch, 1999; Pattison \& Wasserman, 1999; Robins, Pattison \& Wasserman, 1999; Goodreau, 2007; Robins et al., 2007a,b; Fienberg, 2010; Goldenberg et al., 2010; Chatterjee \& Diaconis, 2013; Chatterjee, 2016) have introduced most of the articles up to 2016. There have also been two novel surveys in 2018 (Amati, Lomi \& Mira, 2018; van der Pol, 2018) with a focus on the theory and applications of ERGMs. However, there is a relative paucity of studies investigating ERGMs seminal and new methods together. In addition, to the best of our knowledge, no research has been found examining applications of ERGMs in different fields and contexts in the way that we have done. We believe that this review paper can help the scholars of different disciplines to better recognize the recent applications of ERGMs in their specific field of interest. Certainly, here is still room for more applications of ERGMs in other fields which are yet to be discovered.

There are also some other generative models for network generation such as the use of the neural network for graph generation (Bojchevski et al., 2018; You et al., 2018) and Stochastic ActorOriented Models (Snijders, 1996). However, to the best of our knowledge, ERGMs are one of the oldest methods that have been extensively used in the literature up to now.

Several statistical learning methods have been used for ERGMs parameter learning. In this article, we have addressed the followings:

- Importance sampling

- Stochastic approximation

- Some of the newly presented methods

We have introduced some applications of random graphs in the following categories:

- Medical Imaging

- Healthcare applications

- Economics and management

- Political science

- Missing data and link prediction

- Scientific collaboration

- Wireless networks modelling

- Other applications

Also, some useful tools and libraries have been introduced for the estimation of ERGMs:

- PNet

- R package Statnet

- Bergm

Section 2 is a brief description of the methodology we used to find the articles that we believed are related to the topic of this manuscript. In section 3 we are going to give a formal definition of ERGMs for the readers who are new to this concept. For experienced researchers in the field, this can be used as a refreshment. 
120 Hence, in Section 4, most of the state-of-the-art works for ERGMs estimation methods have been

121

122

123

124

125

126

127

128

129

130

131

132

133

134

135

136

137

138

139

140

141

142

143

144

145

146

147

148

149

150

151

152

153

154

155

156

157

158

159

discussed. Section 5 is a review of ERGMs' applications in multiple fields. In section 6, we have introduced some of the state-of-the-art new libraries and tools for ERGM estimation. Ultimately, in section 7, we conclude what we had said and also give some ideas for future works in the world of ERGMs.

\section{Survey Methodology}

For the purpose of finding related research articles we used two different approach.

1. Searching related keyword in the google scholar search engine.

2. Starting from an initial pool of articles and then move back and forth between their citations and references.

In the first approach we search related keywords like "ERGM", "Exponential Random Graphs", "Exponential Random Graph Models" in the google scholar search engine and extracted related articles by reading their abstracts.

In the second approach which was our main methodology throughout the work we initiate with a number of seminal works which were found by one of the following ways.

1. Being introduced by experts in the field.

2. Extracted from the well-known surveys (Anderson, Wasserman \& Crouch, 1999; Robins, Pattison \& Wasserman, 1999; Pattison \& Wasserman, 1999; Goodreau, 2007; Robins et al., 2007a,b; Fienberg, 2010; Goldenberg et al., 2010; Chatterjee \& Diaconis, 2013; Chatterjee, 2016; Amati, Lomi \& Mira, 2018; van der Pol, 2018) and the well-known book (Lusher, Koskinen \& Robins, 2012).

3. Papers extracted from the first approach which had a good citation count or were published in journals with high impact.

After finding the initial seed of articles by one of the mentioned methods we checked the related publications that they have referenced and the publications that they have been cited from them. We continued until there were no more related articles. In situations which there were too many related articles our selection criteria were mostly based on the citation count and the journals' impact factor.

\section{Precise Definition of ERGMs}

In this section, we give a brief overview of the overall ERGM scheme. According to (Snijders et al., 2006; Robins et al., 2007a), the first work that categorized ERGMs as a separate field of study was (Frank \& Strauss, 1986). Although it was named as Markov graphs at that time, basically it had the same characteristics. An interested reader can refer to (Robins et al., 2007a; Lusher, Koskinen \& Robins, 2012) for more details on both the history and mathematical background of this topic.

In an ERGM, each graph is associated with a probability. This probability indicates the possibility of the presence of that particular graph in the probability distribution of a class of graphs. There are also two other essential elements in ERGMs known as graph configurations and their corresponding parameter. Each configuration or statistics (we will use both names throughout the text) is composed of some nodes and ties repeated in the graph. For example, a triangle consisting

Peer) Comput. Sci. reviewing PDF | (CS-2019:04:37112:1:2:NEW 5 Mar 2020) 
160 of three nodes and edges can be assumed as a configuration. The authors of the seminal work 161 (Frank \& Strauss, 1986) were the first who argued that these configurations can be considered as 162 sufficient statistics for a log-linear mode. Sufficient statistics are features of a i.i.d dataset which 163 are sufficient for modeling the distribution probability of the data such that adding another feature 164 does not add any more insight to the model (RA Fisher, 1922). So, ERGMs are a representation of 165 the graphs by their configurations. A particular exponential function is defined to represent the 166 relationship between these configurations and the probability distribution of the graphs. This 167 formula is a variation of logistic regression which is extended so that it would handle the 168 dependent variable rather than only being applicable to independent variables which are the case 169 for logistic regression (Lusher, Koskinen \& Robins, 2012). We will use the notations presented in 170 Table 1 throughout our work.

171 Note that throughout this work, the representation of the graphs is in the form of the adjacency 172 matrix. For example, in a matrix $x$ if $x_{i j}=1$ it indicates that there is an edge between $i$ and $j$, while 173 if $x_{i j}=0$ no edge exists between these two nodes.

174 Using the introduced notation of Table 1, the ERGM probability function can be expressed as 175 follows:

$$
P(X=x \mid \Theta)=\frac{1}{N} \exp \left\{\theta_{1} c_{1}(x)+\theta_{2} c_{2}(x)+\ldots+\theta_{p} c_{p}(x)\right\}
$$

176 177

178

$N$ is the normalizing factor which is the sum of the probability of all possible graphs computed by Eq. 1, whose formula is as follows:

$$
N=\sum_{x \in X} \exp \left\{\theta_{1} c_{1}(x)+\theta_{2} c_{2}(x)+\ldots+\theta_{p} c_{p}(x)\right\}
$$

If we summarize the results, this leads to:

$$
\begin{gathered}
P(X=x \mid \Theta)=\frac{1}{N} \exp \left\{\Theta^{T} C(x)\right\} \\
N=\sum_{x \in X} \exp \left\{\Theta^{T} C(x)\right\}
\end{gathered}
$$

179 It can be seen in the Eq. 3 that the network configurations are the building blocks of the ERGM

180

181

182

183

184

185

186

187

188

189 formulation. Choosing the correct configurations with the right relation to the network context is central for to the correct estimation of the graphs' distribution. There are two types of network statistics: (1) statistics based on the edge formations, (2) statistics that are based on the node attributes. In the rest of this section, we are going to introduce some basic network configurations (Snijders et al., 2006; Robins et al., 2007a) which have been used in the literature.

Structural configuration refers to the statistics that depend solely on the structure of the graph. Note that their usage is not dependent on the network context and can be applied to any networks. These structures are different in undirected and directed networks.

Some structural configurations that are widely used for undirected and directed graphs are presented in Tables 1 and 2(in Supplemental Files), respectively. 
190

191

192

193

194

195

196

197

198

199

200

201

202

203

204

205

206

207

208

209

210

211

212

Although use of nodal configurations in our model will cause to be more dependent on some specific context, sometimes it is still useful to leverage this kind of network attributes. The reason is that, in many networks, there is a treasure of useful features in the node's metadata and it is not wise to ignore them as one of our model features.

Some descriptions of nodal configurations that are widely used for undirected and directed graphs are reported in Tables 3 and 4(in Supplemental Files), respectively.

According to (Morris, Handcock \& Hunter, 2008), there should not be a linear dependence between the configurations that are used in a model. It is due to that fact that the configurations with linear interdependence with each other cannot add any new benefit to the model and only make the model more complicated.

(Snijders et al., 2006) gave a generalization of ERGMs and also introduced some new configurations. Since then, it has been extensively used in other works. Here we present a brief description of each of them.

Geometrically Weighted Degree Counts (GWDC): this measure is an extension of the nodes' degree combined with geometrically degree discounts in the computation of the statistics, which is expressed as the following expression:

$$
G W D C(x)=\sum_{k=0}^{n-1} e^{-\alpha k} d_{k}(x)
$$

In this equation, $x$ is the matrix we want to compute its corresponding GWDC value and $n$ is the number of nodes in the graph. $d_{k}$ represents the number of nodes with degree $k$. Also, $\alpha$ is a decaying factor which ensures that the nodes with higher degrees have higher impacts.

Geometrically Weighted Stars Counts (GWSC): this measure is an extension of star counts combined with a combination of geometrically degree discounts in computing the statistics, which is expressed as the following expression:

$$
\operatorname{GWSC}(x)=\sum_{k=2}^{n-1}(-1)^{k} \frac{S_{k}}{\lambda^{k-2}}
$$

213 In this equation $S_{k}$ is the number of stars with the $k$ number of edges ( $k$-stars). Also, $\lambda$ denotes a

214 decaying factor which ensures that the stars with a higher degree have a greater impact.

215 Sum of Ascending Factorial Degrees (SAFD): first presented in (Handcock \& Jones, 2004), it is a 216 variation of Yule distribution using the sum of ascending factorials of degree ${ }^{1}$ :

$$
\operatorname{SAFD}(x)=\sum_{i=1}^{n} \frac{1}{\left(y_{i+}+c\right)_{r}}
$$

217 Transitivity by Altering $k$-Triangles (TAT): this measure is an extension of triangle counts 218 combined with geometrically discounts in the computation of the statistics, which is expressed as 219 the following expression:

$1(d)_{r}=d(d+1) \ldots(d+r-1)$ 
220

221

222

223

224

225

226

227

228

229

230

231

232

233

234

235

236

237

238

239

240

241

242

243

244

245

246

247

248

249

250

251

252

253

254

$$
\operatorname{TAT}(x)=3 T_{1}-\frac{T_{2}}{\lambda}-\frac{T_{3}}{\lambda^{2}}-\ldots+(-1)^{n-3} \frac{T_{n-2}}{\lambda^{n-3}}
$$

In this equation, $T_{k}$ is the number of $k$-triangles. $\lambda$ represents a decaying factor which ensures that the triangles with a higher degree have a more substantial impact. Figure 2 (in Supplemental Files) displays a description of $k$-triangles.

Altering Independent Two-Path (AI2P): this measure is an extension of 2-path with a combination of geometrically discounts in the computation of the statistics, which is expressed as the following expression:

$$
A I 2 P(x)=U_{1}-\frac{2}{\lambda} U_{2}+\sum_{k=3}^{n-2}\left(\frac{-1}{\lambda}\right)^{k-1} U_{k}
$$

In this equation, $U_{k}$ is the number of star $k$-independent 2-paths. $\lambda$ represents a decaying factor which ensures that the triangles with higher degrees have higher impacts. In Figure 3 (in Supplemental Files), you can see a description of $k$-independent 2-paths.

The authors of (Wilson et al., 2017) addressed one of the significant drawbacks of ERGMs. As can be seen in Tables 2 and 3, the weights of the graphs are missing. In other words, they are only applicable to unweighted graphs, and if we want to use them in the context of the weighted graphs, their weights should be omitted. However, much useful information underlies the weight of the graphs and for most of the domains it is crucial to consider them to accurately model the graph. Following this idea previously discussed in (Desmarais \& Cranmer, 2012; Krivitsky, 2012), they continued to design more flexible estimation methods for the so-called Generalized ERGMs (GERGM). Their method can handle a wide range of graphs' statistics with continuous-valued edges.

The endogenous statistics need to be selected before implementing the model. Therefore, there must be several assumptions about choosing a particular statistic. Although the process of finding the best statistics for the model is highly empirical, considerations when making a hypothesis about the network's configurations is important. The choice of a specific statistic is highly dependent on the assumptions we have about network phenomena. Simple structures like the number of edges and nodes take control of the size and sparsity of the graph. In a friendship network, triangles can indicate the inclination of mutual friends becoming friends with each other. In a citation network stars refers to a large number of central nodes (van der Pol, 2018). The dyadic dependence assumption between nodes should also be considered while choosing the proper statistics for the model. Dyadic dependence is the dependent processes among two dyads. A dyad in this context refers to a pair of nodes and their relation. The dyadic dependence among processes could arise a number of problems like model degeneracy, for more information see (Handcock et al., 2008). New specifications like geometrically weighted degree counts and Altering $k$-Triangles have been introduced to alleviate model degeneracies resulted from dyadic dependence. This is achieved by 
255

256

257

258

259

260

261

262

263

264

265

266

267

268

269

270

271

272

273

274

275

276

277

278

279

280

281

282

283

284

285

286

287

288

289

290

291

292

293

increasing the stability of the model with weighting the low density and reducing the weight for higher degrees to avoid the degeneracy (Snijders et al., 2006; van der Pol, 2018).

\section{Methods for Estimation}

One crucial step in the ERGMs models is to fit the coefficient of the model to the observed data after designing the model with desired configurations. Multiple methods exist for this purpose. Nevertheless, the overall approach in all of them is developing a likelihood function based on the ERGM formulation and then solving it with some of the mathematical methods that exist for Maximum Likelihood Estimation (MLE). Note that all of the MLE solution methods should be specialized for the ERGM modeling. After introducing the general form of the mentioned likelihood function, we are going to present a brief description of some of the methods for solving it already presented in the literature.

\section{A form of the likelihood function}

We aim to find the best values of the $\Theta$ vector in Eq. 3 which maximize the probability over the observed data. In a more formal expression, we want to solve the following equation:

$$
\Theta_{M L}:=\operatorname{argmax}_{\Theta \in R^{k}} P(X \mid \Theta)
$$

Where, $P$ is the same probability function as the Eq. 3 and, $R^{k}$ represents all possible real values over a $k$-dimentional space. Note that the $\Theta$ is a vector of coefficients rather than a single value; thus, its space value should be a vector space. Different methods exist for solving such equations. Here, we are going to name a few of them which are mostly used in the ERGM related works. Also, we intend to present a number of state-of-the-art methods that have been presented after 2016.

\section{Preliminaries}

\section{Sampling methods}

There are two important applications of sampling methods in the ERGMs parameter estimation model:

- In all methods, there is a need to simulate graphs from the fitted model or simulate some graphs to gain more insight into the distribution of the graphs and their configurations (Lusher, Koskinen \& Robins, 2012). This distribution is also used to test whether the distribution of the fitted model is close to the observed data or not.

- Predicting the prior distribution of the graphs for Bayesian learning models

So, there is a need for sampling methods to draw a sample from the given graph distribution. In this section, we present some of the sampling methods that have been used extensively in the literature.

Monte Carlo Markov Chain sampling method which is abbreviated to MCMC (Metropolis et al., 1953) is a well-known sampling method which has been used in many works. Here, we only discuss it in the context of graph generation. In this method, we start with an initial graph which can also be an empty graph. Then, in each iteration, a new graph is generated by making a small change to the graph from the last step. The form of this "change" is different from work to work. The most straightforward change is adding or removing a tie. The procedure is as follows: two nodes are chosen randomly. After which the state of their connection is altered (if they are already 
294

295

296

297

298

299

300

301

302

303

304

305

306

307

308

309

310

311

312

313

connected, they become disconnected while if they are not connected, they become connected.). In the next step, the probability of the generated graph is computed according to Eq. 3. This probability is compared to that of the graph generated in the previous step. Then, we accept or reject the new graph based on the comparison of these two probabilities. If the new graph is more probable, it is more likely to substitute the old graph in the next iteration. The probability of whether the new graph is chosen for the next iteration or the graph from the last step is re-chosen depends on which one of them has a higher probability score in Eq. 3. Note that only having a higher probability score is not a guarantee that the graph gets chosen. It only increases the chance of selection. All these outlined the scheme of all MCMC methods. However, the details including how many of ties are altered in each iteration or the probabilistic selection between the old graph and the new one are different in literature. We intend to present a quick introduction to the Metropolis-Hasting sampling methods which is mostly used in ERGM related literature. Figure 1 displays the overall procedure of an MCMC method.

Metropolis-Hasting (Metropolis et al., 1953) is the most widely used MCMC derivation in ERGM studies. Metropolis Hasting in the context of graph generation is as follows. Initially, as we explained in the general MCMC scheme, we start with an empty or random graph. Our goal is to generate $N$ samples from the distribution of graphs, implying that we want to generate a sequence of $x_{1}, x_{2}, \ldots, x_{N}$ graphs. We choose two random nodes at each step and change the tie situation between them. The probability of the newly generated graph and the graph from the last step is then computed using the following formula:

$$
\min \left\{1, \frac{P\left(X=x_{n e w} \mid \Theta\right)}{P\left(X=x_{N-1} \mid \Theta\right)}\right\}
$$

314 This formula computes the probability of whether to accept the new move or substitute the last 315 step graph as the new one.

316 Classic methods

317 So far, we have reviewed the necessary preliminaries. Now, we can review the most widely used 318 methods in the literature for estimating the value for statistical parameters $(\Theta$ in Eq. 4) best 319 representing the observed data. In other words, our aim is to solve Eq. 10. Most of the methods 320 use the following steps: Initially, they start with an initial value for the parameter vector. Then, the 321 distribution of the graphs is generated by one of the sampling methods. Next, the difference 322 between the distribution and the observed data is computed $\left(E_{\Theta}(C(X))-C\left(X_{\text {observed }}\right)\right)$. If the

323

324

325

326

327

328

329

330

331 difference is satisfactory, the learning process is halted and the current vector of the parameter is considered as the final answer which best fits the observed data; Otherwise, based on the learning method the algorithm moves to the subsequent values of $\Theta$ and goes back to step 2. Figure 2 demonstrates the finite automata of this method.

The ultimate goal of all learning methods is to find a vector of $\Theta$ values in Eq. 3 that can also generate graphs which are similar to the observed graphs. To this end, different learning methods exist and this section describes the most important of them namely importance sampling and stochastic approximation. We used the description presented in (Lusher, Koskinen \& Robins, 2012). 
332

333

334

335

336

337

338

339

340

341

342

343

344

345

346

347

348

349

350

351

352

353

354

355

356

357

358

359

360

361

362

363

364

365

366

367

368

369

370

371

\section{Importance sampling}

The goal as we said is to minimize the expected value of $\Theta$ which minimizes the expected value between observed statistics and the ones generated by the ERGM model. The aim is to use Maximum Likelihood that we discussed before to find the best value of vector $\Theta$ which maximize the right hand side of Eq. 10. One possible approach is to search over all possible $\Theta$ values in the search space and try them one by one. But since the search space is very large and $\Theta$ values are continuous this approach is not practical. Instead of such brute force algorithm, one of the methods for ERGMs parameter estimation is the one inspired by the general framework ML estimation method for dependent data introduced by (Geyer \& Thompson, 1992). The main idea is to instead of generating the whole possible graphs of a particular $\Theta$ vector, we can draw a large sample of the graphs and consider it as a representation of the whole possible graphs at each iteration. This sample is generated from the current value of the $\Theta$ vector using the Eq. 3 and is used in the formula to compute $E_{\Theta}(C(X))$ and then compute how much the value $E_{\Theta}(C(X))-C\left(X_{\text {observed }}\right)$ is close to zero. At each iteration an average over the generated graphs statistics is computed to measure $E_{\Theta}$ $(C(X))$ and decide whether to continue the estimation or not based on how much the $E_{\Theta}(C(X))-C$

$\left(X_{\text {observed }}\right)$ is close to zero. Other than the mentioned halting situation we need an algorithm to move from each $\Theta$ vector to a new one (if the halting is not satisfied). A Newton-Raphson formula is used to move from one statistic to another. For more detail on the mathematical details of the sampling and the used Newton-Raphson based method see (Lusher, Koskinen \& Robins, 2012).

\section{Stochastic approximation}

The This model presented in (Snijders, 2002) can handle both bimodal and multimodal and enhance the speed of convergence. They also used the Newton-Raphson method for the learning step of the algorithm. As mentioned in (Lusher, Koskinen \& Robins, 2012), they used a three-step method. At phase one, a limited number of iterations is performed to determine initial values of the algorithm. In the second step, the Newton-Raphson algorithm is employed to optimize the answer. Finally, the convergence criteria are checked.

\section{Newly presented methods for ERGM estimation}

In (Byshkin et al., 2016), the authors improved the MCMC sampling part of the ERGM estimation by adding an auxiliary parameter to the model. In their method, which they called Improved Fixed Density (IFD) MCMC sampler, they tried to decrease the state space of the network to reduce the time complexity of the algorithm. This new auxiliary variable which was based on the number of ties helped the model to converge faster without the need of making the MCMC overall model more complicated.

In some works like (Stivala et al., 2016), snowball sampling (Coleman, 1958; Goodman, 1961) was used to overcome the computational complexity of the MCMC method over large network datasets.

As mentioned earlier, the Bayesian estimation of the parameters requires prior knowledge about the network posterior distribution. However, this posterior probability distribution is not always easily available. To overcome this issue, (Bouranis, Friel \& Maire, 2017) introduced a pseudolikelihood estimation approach by replacing the posterior distribution with a more achievable 
372 pseudo-distribution. Although this method resulted in faster computation of the likelihood 373 function, as mentioned in the (Schmid \& Desmarais, 2017), its results are not still as precise as 374 they should. To handle this problem, the same mentioned article introduced another pseudo375 likelihood estimator based on the bootstrapping parameters which culminated in more accurate 376 convergence.

377 In a recent work (Bouranis, Friel \& Maire, 2018), the authors proposed yet another heuristic model 378 based on pseudo-likelihood estimation. They did so by performing three adjustments to the 379 pseudo-likelihood function: 1) mode corrections to overcome the bias of the pseudo-likelihood 380 function; 2) curvature adjustment, which is a modification in the selection of the transformation

381

382

383

384

385

386

387

388

389

390

391

392

393

394

395

396

397

398

399

400

401

402

403

404

405

406

407

408 matrix and the corresponding Hessian matrix; and 3) magnitude adjustment, which is a linear transformation to scale the curvature-adjusted pseudo likelihood to the right values.

Despite all the progress in the ERGMs parameter estimation and modeling, it is still a hard task in large graphs. (Thiemichen \& Kauermann, 2017) addressed two of the main challenges of ERGMs, including the instability of the model especially in the models with more straightforward statistics like triangles and the time-consuming nature of the ERGM parameter estimation procedure due to large number of numerical simulations. For solving the first problem, they proposed a technique to produce smooth stable statistics. Further, to overcome the second issue, they employed a novel subsampling model which instead of fitting the model to the whole network it only fit the model to subgraphs from the network and then aggregated these sample estimates. The two mentioned ideas yielded a significant improvement for modeling large graphs.

\section{ERGMs variations}

Apart from the basic definition of ERGMs there are also some other variations of ERGMs. Each year a number of new extensions of the original ERGM definition are introduced. In this chapter we introduce three of the most widely used ERGMs variations.

Evolution of networks in dynamic environment like social networks has attracted scientist to make an extension of the ERGMs called Temporal ERGMs a.k.a. TERGMs which is capable of capturing the information underlying dynamics of such networks (Hanneke et al., 2010). A Markov assumption between snapshots of the network at each timestep is taken. Then the model is created based upon the relation between each two consecutive snapshots $S_{t}$ and $S_{t-1}$.

$$
P\left(X=S_{t} \mid S_{t-1}, \Theta\right)=\frac{1}{N\left(\Theta, S_{t-1}\right)} \exp \left\{\Theta^{T}, \psi\left(S_{t}, S_{t-1}\right)\right\}
$$

As it can be seen in Eq. 12 most parts of the formula for TERGM is similar to normal ERGM. However, the time snapshots are now considered and each new time snapshot $S_{t}$ is dependent to its previous one $S_{t-1}$. Also, the normal count of the networks statistics has been substituted with temporal potential count $\psi$ over two consecutive snapshots. For more information see (Hanneke et al., 2010) and for the information about the btergm which is a library for temporal ERGMs see (Leifeld, Cranmer \& Desmarais, 2017).

Most of the real-world network are associated with a value on their edges which are referred to as weighted graphs in graph theory. A plethora of researches have been done to consider these types 
409

410

411

412

413

414

415

416

417

418

419

420

421

422

423

424

425

426

427

428

429

of networks into the ERGM general schema. GERGM (Desmarais \& Cranmer, 2012) and the model proposed by (Krivitsky, 2012) are the two most well-known models which have incorporated the networks' edges' weights into the model. The normalizing factor in Eq. 3 which is the denominator of Eq. 4 is not assured to be convergent when the network statistics $(C(x)$ in the Eq. 4) are infinite set like continuous valued edges. GERGM is a model aimed to overcome this issue by using a probability model for such continuous values. They build a transformed version of the original ERGM formula that no longer suffers from the mentioned problem. The (Krivitsky, 2012) have also extended the previous binary version of ERGM which only models edges existence rather than their value into a model which is capable of capturing the information of weighted graphs. However, his method is restricted to natural valued weights on the edges.

In network science there is a special kind of networks called multiplex or multilayer networks. These are networks which their nodes are connected in the context of more than one attribute. For example, in a social relation network, actors might have several relations between them like friendship network or co-working network. Each of these relations can be abstracted as a layer in a network model. Also, in some situations, there is a hierarchical structure in the data like modeling the relations inside a university. There are schools, which are divided into groups and lecturers and students. An extension of ERGM which is applicable to model such scenarios in multilevel networks is proposed for these networks (Wang et al., 2013). They considered relation between the nodes in each level and also the inter-level relations into the model. For example, consider a two layer network with layers $A, B$ and an imaginary layer between them called $x$ which is for the purpose of modelling inter-level relations between $A$ and $B$. Then the Eq. 1 is re-written as:

$$
\begin{aligned}
& P(A=a, X=x, B=b \mid \Theta) \\
& =\frac{1}{N} \exp \left\{\Theta_{a}^{T} C_{a}(a)+\Theta_{b}^{T} C_{b}(b)+\Theta_{x}^{T} C_{x}(x)+\Theta_{a, x}^{T} C_{a, x}(a, x)+\Theta_{b, x}^{T} C_{b, x}(b, x)+\Theta_{a, b, x}^{T} C_{a, b}\right)
\end{aligned}
$$

430

431

432

433

434

435

436

437

438

439

440

441

442

443

444

Which the $\Theta_{a}^{T}, \Theta_{b}^{T}, \Theta_{x}^{T}, \Theta_{b, x}^{T}, \Theta_{a, x}^{T}, \Theta_{a, b, x}^{T}$ are the parameters for statistics which are extracted from layers $a, b$ and the inter-level relations $a, x$ and $b, x$ and the inter-level relation of layers $a, b$. The same is true for the count functions of the statistics. $\Theta$ is the set of all types of statistics.

\section{Applications of ERGMs}

As mentioned previously, ERGMs are a useful tool for scientists from various disciplines. Networks are everywhere, and anywhere that they exist they can be analyzed using ERGMs and other statistical models. Note that here we have mostly reviewed the works since 2016.

\section{Medical Imaging}

In order to take care of the limitations of the descriptive analysis of brain neural networks, the author of (Sinke et al., 2016) used ERGMs to be able to model the observed network using the joint contribution of network structure. They also compared the changes in brain networks statistics across different ages. This study was conducted to examine the effects of aging during lifetime in the brain global and local structures. Graphs where extracted from brain images obtained from diffusion tensor imaging (DTI). Four network statistics were used to model these networks: 
445

446

447

448

449

450

451

452

453

454

455

456

457

458

459

460

461

462

463

464

465

466

467

468

469

470

471

472

473

474

475

476

477

478

479

480

481

482

483

484

- The number of edges

- The geometrically weighted edgewise shared partner (Hunter, 2007)

- The geometrically weighted non-edgewise shared partner (Hunter, 2007)

- The hemispheric node match: a binary indicator which shows whether two nodes are in the same hemisphere of the brain.

The Bayesian learning schema from (Caimo \& Friel, 2011) was used to fit the model.

In a recent work, (Dellitalia et al., 2018) employed ERGMs to study the structure of neural networks of the brain. They aimed to increase the chance of unconscious and injured patients to recover by analyzing brain functional data. In their work, they overcame four shortcomings of previous methods by incorporating ERGMs into their study. For example, one of them was the ability to assess the dynamics of the network over time. They used the Separable Temporal ERGMs (TERGM) (Krivitsky \& Handcock, 2014) for their modeling. One of the aspects of their work that successfully handled with ERGMs was that the network structures they chose should have not been necessarily independent. This restriction was one of the main drawbacks of previous methods. Functional Magnetic Resonance Imaging or fMRI is a method for observing brain activities and their changes over time. There are components in the fMRI images which can be explained using network analysis methods. Nodal signals, network architecture, and network function are the three essential network properties in building fMRI-based networks (Solo et al., 2018). ERGMs are one the main important network analysis methods which have been used to explain such networks. The authors of a review paper (Solo et al., 2018) introduced the most critical efforts with the aim to explain these brain networks. Note that there are plenty of works which used ERGM as their method (Simpson, Hayasaka \& Laurienti, 2011; Simpson, Moussa \& Laurienti, 2012).

\section{Healthcare Applications}

Having a healthy life is one the central concerns of human life. If we look at this issue from a macro perspective, we can see that many health-related problems can be alleviated by analyzing their corresponding inter-related actors. For example, in epidemiology, there is a direct connection between the patient relationships and the extent that the disease can spread. In most cases, these relations between the actors will result in the formation of a network. This network can be analyzed using ERGMs to answer different questions underpinning its formation and dynamics. This kind of analysis is something that has already been done extensively by researchers in the healthcare community.

Analyzing inter-hospital patient referral network is a significant problem which (Caimo, Pallotti \& Lomi, 2017) has recently investigated using ERMGs. They used a combination of the edges and nodes of the network and utilized the Bayesian approach introduced in (Caimo \& Friel, 2011) to fit their model. This task was done using BERGM (Caimo \& Friel, 2014) R language package for their implementation.

Another work (Baggio, Luisier \& Vladescu, 2017) shed light on the relationship between social isolation and mental health. The connection between these two subjects was investigated by analyzing the network of Romanian adolescents using ERGM modeling. They concluded that there is a strong link between the two mentioned concepts.

Peer] Comput. Sci. reviewing PDF | (CS-2019:04:37112:1:2:NEW 5 Mar 2020) 
485 Application of statistical network in epidemiology and disease spreading is another interesting 486 topic which has attracted from the attention of the biological science community. (Silk et al., 2017) 487 provided an important opportunity to advance the understanding of the pattern and evolution of 488 infections in static and dynamic environments. They also used ERGMs for their models. In their 489 ERGM model, they employed a fair number of both structural and node-based attributes. The 490 ERGM (Hunter et al., 2008) R language package was used for the tests.

491 Social ties can reveal a wide range of aspects of human life. The networks formed by such ties and 492 edges among individuals can transfer life habits and behaviors in a society. For example, in many 493 kinds of literature, the relationship between social tie and analysis of obesity has been investigated. 494 (Zhang et al., 2018b) thoroughly studied the articles related to applications of social network 495 analysis to obesity. In another work related to eating disorders, (Becker et al., 2018) have presented 496 some findings using ERGM network analysis about the relationship between the eating disorders 497 and human relationships. They conducted their study on members of a sorority at Southeastern 498 University.

\section{Economics and Management}

500 Marketing organizations that are responsible for promoting tourist destination have also been 501 analyzed using ERGMs. (Williams \& Hristov, 2018) intended to study the networks underpinning 502 Destination Marketing Organizations (DMOs). They developed four models with the most 503 complex one consisting of the following statistics:

504

505

506

507

508

509

510

511

- Number of edges

- The geometrically weighted edgewise shared partner (Hunter, 2007)

- Properties of membership and industry background

Global migration and different attributes of immigrants can be considered as a network. There are many theories on how these networks shape and evolve and how they depend on immigrants and country backgrounds. ethnicity, wealth, religion). (Windzio, 2018) applied ERGM in order to examine theories and hypotheses about creation and evolution of these networks. He used both the graph structure and node attributes in a large number of statistics.

512 Global tourism and its corresponding network, Global Tourism Network (GTN), is yet another 513 field of study, given the tremendous financial importance of tourism market. As mentioned in 514 (Lozano \& Gutiérrez, 2018), it is essential to gain insight into the connections between its 515 components. In the same article, an ERGM approach was used to find the critical local 516 substructures of the GTNs.

517 Handling the budget and resources during crises is always a challenging task for humanitarian 518 organizations. There is a need for a tradeoff between the use of asset supplies for the current crises 519 and the usual ongoing projects. This problem has been formulated in the form of asset supply 520 networks. (Stauffer et al., 2018) used ERGMs as an empirical model to understand the asset flows 521 during a crisis.

522 The applications of ERGMs have even been extended to the analysis of online drug distribution 523 networks. In a recent work, (Duxbury \& Haynie, 2018) conducted the mentioned research on a 524 dataset of an online drugstore on the dark web. They studied such networks concerning their 
525 topological dynamics, suppliers, and customer demand as well as the resistance of such networks

526 to disruptions.

527 Does economic partnership between professionals will result in further trust and solidarity? This

528 is the central question of (Bianchi, Casnici \& Squazzoni, 2018). They developed an ERGM

529 multiplex network model collaboration network and a number of other attributes and then analyzed

530 it using multivariate ERGMs to examine social support and trust for each of the network statistics.

531 Political Science

532 A large number of articles in the political science community have used ERGMs for their

533 modeling. This enthusiasm toward ERGMs among political science scholars well suggests that it

534 is among the most famous mathematical modeling in the field. Here we introduce a handful of

535 these articles.

536 Sustainable development policy is a major concern both for the government and the private sector.

537 It is only achievable by interaction among individuals. In particular, the role of the connection

538 between funding sectors and those in need of money is important for carrying out their projects.

539 This is the central problem of (Gallemore \& Jespersen, 2016). The dataset consisted of 91 donor

540 organizations. The role of ERGM in this work was modeling the donor agent relationship

541 networks.

542 Another major issue that has been addressed through ERGMs is collaborative governance between

543 different sectors and individuals of multiple organizations. In (Ulibarri \& Scott, 2016), the authors

544 used ERGM to test their hypothesis about what should be observed in low-collaboration vs. high-

545 collaboration networks. Four simple ERGMs' configurations were used, including:

546 - The number of networks ties

547 - The number of nonzero ties

548 - The number of reciprocity relations in the network

549 - The number of transitivity relations in the network

550 In a more recent work, (Scott \& Thomas, 2017) addressed the same problem. However, they used 551 different datasets and network statistics. (Hamilton \& Lubell, 2018) also took the same ERGM

552 modeling approach in discussing the collaborative governance, in the special domain of climate 553 change adoption.

554 In an exciting work, (Li et al., 2017) investigated the effectiveness of military alliances in making 555 peace between states. They used temporal random graph models for longitudinal network data of 556 alliance. They employed two different sets of network statistics and developed two models upon 557 them.

558 Communications via internet social networks have helped the human to take a huge step further. 559 People from multiple backgrounds and societies are engaged in conversations that have never been 560 possible before the widespread popularity of online social networks. In the case of political 561 conversations in social networks, there is always the dilemma whether this freedom has resulted 562 in more communications between people with different ideologies or adversely it will cause people

563 with same viewpoints tend to dominate most of the conversation thereby self-reinforcing the same 564 way of thinking. (Song, Cho \& Benefield, 2018) addressed this issue by studying the network of 
565

566

567

568

569

570

571

572

573

574

575

576

577

578

579

580

581

582

583

584

585

586

587

588

589

590

591

592

593

594

595

596

597

598

599

600

601

602

603

604

message selection of users during a presidential election and then analyzed the mentioned network by a Temporal ERGM (TERMG) to answer the questions above. The world trade network has also been investigated via TERGMs. (Pan, 2018) studied these networks to answer the underlying questions about them and their effects on related subjects.

Even further, some works such as (Chen, 2018) took the use of ERGM networks in modeling political networks a step further by incorporating multilayer networks properties into their models. He proved with experimental results that this multilayer approach toward ERGMs could better fit the model to the observed data.

The analysis and challenges of power transition in a personalized authoritarian system is a problem that has been discussed in (Osei, 2018) using ERGM modeling. In addition to qualitative methods, the author employed ERGM as a quantitative method to answer questions about the regime survival of the regime under the mentioned situations. The network in this context consisted of elite interactions network in authoritarian countries. They found that many of the important people in the past ruler administration still play a crucial role in the current government.

Environmental treaties among governments play a vital rule in solving environmental issues. However, coming to an agreement in such commitments is not straightforward. The aim of (Campbell et al., 2019) is to study the model of ratification in such treaties among different parties or states. The main contribution of this research is to find out how the influence network between countries can affect the interdependency of countries decisions on environmental politics. To this end, they have used Bipartite Longitudinal Influence Network (BLIN) model to extract two latent influence network using which show negative and positive influence among different countries. Later these two networks have been analyzed using ERGMs to find the effective contextual and structural network statistics on the shaping of influence (negative or positive) networks (Marrs et al., 2018).

Network of international arm trade is yet another subject that has been studied using ERGM simulations (Thurner et al., 2019). The structure of weapon exchanges network between countries and alias is very complicated. A plethora of effective factors are effective in the formation of the network. Economic enhancement of the seller and the desire to strengthen they allay in different regions of the words are two important considerations from the dealers. Their datasets are extracted from available data of arm deals after world war II. Temporal ERGMs were used for during the analysis. They have used a number of statistics based on their hypothesis about importer and exporter effects, size of the countries' domestic economic markets, national material capabilities, conflict involvement joint membership in defense agreement, geographic distance between two countries. Most of the statistics used in this work were exogenous statistics.

\section{Missing data and Link prediction}

Link prediction is the problem of finding missing links in a network. As we explained, ERGM deals with estimating graph distribution and generating a new graph based on them. Graph generation part is the exact process of finding missing links. However, in link prediction, we do not want to estimate the whole graph distribution, and we desire to find the probability of link formation between two nodes based on the current structure of the graph.

Peer] Comput. Sci. reviewing PDF | (CS-2019:04:37112:1:2:NEW 5 Mar 2020) 
605 (Smith, 2012) used ERGMs to create a global view of networks with missing data based on 606 sampled data. In their approach, they took sampled ego networks and tried to estimate features of 607 the whole network. The interesting fact about this work was that not both the structure of the 608 network and its size were unknown. A three-step algorithm was used, and in the last step, the aim 609 was to predict the global structure of the network from the fitted model. Two real-world network 610 data were used in the tests including addition of health network and sociology co-authorship 611 network.

612 (Koskinen et al., 2013) used the same approach of leveraging ERGM for data augmentation in 613 graphs with missing tie variable. In an empirical test, they were able to estimate the missing tie 614 variable of a network with 74\% missing tie with fair precision. As the article name suggests, they 615 used a Bayesian estimation method for fitting the parameters of their model.

616 (Zhang, Zhai \& Wu, 2013) applied ERGMs for predicting links in microblogs. They used five 617 kinds of graph statistics with four of them (2-5) introduced in (Hunter, 2007):

- Number of edges

620

621

622

623

624

625

- Gwidegree (Geometrically weighted indegree): the weighting indegree of the network.

- Gwidegree (Geometrically weighted outdegree): the weighting outdegree of the network.

- Gwodegree (Geometrically weighted dyadwise shared partner): the number of shared

627

628 nodes of all node pairs in the network.

- Gwesp (Geometrically weighted edgewise shared partner): similar with Gwdsp, it is the number of shared nodes for linked node pairs in the network.

The link prediction based on the ERGM method introduced in this article is an iterative approach. At each step, they compute the conditional probability of adding an edge between two arbitrary nodes having the observed part of the network. This process is performed several times through an MCMC simulation, and at last the average of all these steps is computed:

$$
P\left(X_{i j}=1 \mid X^{c}=x^{c}\right)=\frac{1}{N} \exp \left(\theta^{T} C\left(x_{i j}=1, x^{c}\right)\right)
$$

629

630

631

632

633

634

635

636

637

638

639

640

641

In Eq. 14, $X_{i j}$ is the probability of presence of an edge between nodes $i$ and $j . X^{c}$ is also the state of all other edges in the time predicting $X_{i j}$.

Five datasets have been used:

- Sina Microblog dataset community of "Beijing badminton community."

- Sina Microblog dataset community of "Beijing bicycle community."

- Sina Microblog dataset community of "Data mining community."

- Scientist co-authorship dataset GR (General Category)

The authors of (Krause \& Caimo, 2019) have presented a new estimation algorithm for Bayesian Exponential Random Multi-graphs model which is an imputation model applicable to such multilayer networks. This work is an extension of the (Koskinen, Robins \& Pattison, 2010) to multilayer networks.

An interested reader can refer to a recent survey on different imputation method on network missing data (Krause et al., 2018). One of the advantages of this methods is that it is solely about 
642 missing data in the context of networks. Different missing data treatment methods have been tested

643 on different missing data in a complete benchmarking framework.

644 Scientific Collaboration

645 Finding the best colleagues or best-related research papers and topics is always a significant issue

646 for anyone in the scientific community. Co-author and citation networks analyses are two

647 important topics that have been extensively studied in research related to analysis of networks

648 addressing these issues.

649 The researchers in (Zhang et al., 2018a) addressed the effect of three major network properties in

650 scientific collaboration networks including Homophily, Transitivity, and Preferential attachment.

651 Performing an ERGM study on these networks, they argued that incorporating the mentioned

652 properties we can provide more insight into how collaborations form. The data for this study were

653 collected using the metadata of papers' citations from the Web of Science from 1956 to 2014.

654 As we approach more complex scientific phenomena, we more feel the need for collaboration

655 between different scientific communities. (Fagan et al., 2018) also studied a co-authorship network

656 to evaluate the changes in inter-disciplinary scientific articles. More precisely, they applied a

657 special form of ERGMs called the Separable Temporal ERGMs (STERGM) (Krivitsky \&

658 Handcock, 2014) to evaluate the co-authorship network over time and make prediction ties in the

659 network. They employed some structural and nodal attributes. Structural attributes refer to a

660 number of edges, degree, and triadic closure, while some nodal attributes capture whether two

661 individuals have the same professor rank, gender, and college.

662 ERGMs are widely used for citation networks analysis. (An \& Ding, 2018) performed the same 663 study in the special case of publications on causal inference. They argued that some technical and 664 social processes are underpinning citation networks. Their ultimate goal was to explain the 665 essential factors in forming a citation network and predicting the citation patterns.

666 An in-depth study of polarization among researchers of the field of social science was performed 667 in a recent work (Leifeld, 2018). He used both qualitative and quantitative methods to address the 668 most compelling reasons and strategies causing the polarization. He applied ERGM as his 669 qualitative method over two co-authorship networks in the field of social science in two separate 670 countries.

671 Other than the studies on co-authorships and citation networks there are other aspects of scientific 672 collaboration that have been widely studied. One of such studies is the study on how the 673 recruitment of new members of scientific collaborators in scientific organizations takes place. In 674 a study (Leifeld \& Fisher, 2017) the dynamics underlying the membership procedure of new 675 scientist in international scientific assessments has been evaluated. The authors have used a dataset 676 extracted from an international well-known research program on world's ecosystem called 677 Millennium Ecosystem Assessment (MA). Their method is based on analyzing the pattern of the 678 network formation by ERGM using a number of exogenous and endogenous network's statistics. 679 The analysis approved the authors hypothesis which suggests that factors like having the same 680 nationality to the previous researcher in the research group or being in the same institution with 681 them have a high impact on the recruitment of new researchers. This could result in lack of 682 diversity of opinions in the final outcomes of the assessments conducted by the research group. 


\section{Wireless Networks Modelling}

684 Random Geometric Graphs also known as RGGs are defined as the group of graphs which are

685 obtained by placing a number of nodes randomly in a geometrical space and draw vertices between 686 those nodes which their distance is less than a threshold $d$ in a given norm (Penrose $\&$ others, 687 2003). One issue in the wireless sensor networks is that there is not a fixed placement for the 688 nodes in most of times. The nodes are randomly distributed and therefore the shape of connecting 689 graph tend to be very volatile (Raghavendra, Sivalingam \& Znati, 2006). Studying these graphs 690 formation and the statistical dynamic behind their formation has been extensively investigated in 691 the literature related to RGGs (Iyer \& Manjunath, 2006). For example, exponential RGGs which 692 are the RGGs that the distribution of their nodes is also exponential (Gupta, Iyer \& Manjunath, 693 2008). These graphs have been used for modeling wireless sensor networks (Shang, 2009; 694 Kenniche \& Ravelomananana, 2010). In the (Shang, 2009) the wireless sensors are assumed to be 695 on a line and to evolve over time with respect to a dynamic RGG process. The effect of statistical 696 properties for a particular time snapshot has also been considered in this paper. Such analysis with 697 using one-dimensional RGG has also been done in the past in (Karamchandani et al., 2006). 698 Vehicular Ad Hoc Network are yet another use case for RGGs (Zhang et al., 2014). Due to the 699 movement of the vehicles and the rapid changes in the graph they have many similarities to 700 previous applications of RGGs.

\section{Other Applications}

702 The applications of ERGMs are so extensive that some works cannot be organized in a particular 703 category. In this section, we introduce some of them.

704 The concept of social networks is not limited only to human relationships. There are some complex 705 interactions in animal behaviors which can be modeled as graphs. ERGMs are also a useful tool 706 for analyzing these kinds of networks. In a recent work, (Silk \& Fisher, 2017) reviewed the use of 707 ERGMs in such studies. Also, more specifically, other recent works have leveraged ERGMs 708 capabilities in their specific context. (Hellmann \& Hamilton, 2018) is a work in which the authors 709 investigated the effect of neighbors' mediation in cooperative fish breeding by analyzing their 710 interactions with an ERGM model. In another work (Silk et al., 2018), the same approach was used

711 to investigate sex-related disease spreading through animal contact networks in three sorts of 712 animal networks.

713 In a novel work, (Müller, Grund \& Koskinen, 2018) studied the social inequalities in Sweden by 714 analyzing an immigrant movement flow network on both the micro and macro levels. Their 715 network was a directed binary graph with Stockholm's neighborhoods as the nodes and ties as the 716 representative of the movement flow across neighborhoods. Only structural features (statistics) 717 were used in ERGMs.

718 How do networks respond to a sudden change? Which sort of disruptions is most influential in the 719 network upcoming status? How the network will react to a change or what is the best reaction? 720 These are all questions that can be summarized as "forecasting social network reaction to 721 disruption." In a recent article,(Mellon \& Evans, 2018) reviewed state-of-the-art research articles 722 concerning these topics in various fields. According to them and by mentioning one of their 723 previous works (Mellon, Yoder \& Evans, 2016), ERGMs can play a crucial role in this issue. In 
724 the mentioned work, they used ERGMs to examine the network formation mechanism before and

725 after the intervention. According to their findings, networks tend to preserve these mechanisms 726 following the disruptions.

727 Tools and Libraries

728 There are a number of useful tools and libraries that facilitate use of ERGMs in different domains.

729 PNet and its extension for multilevel networks (MPnet) and bivariate analysis (XPnet) were 730 introduced by (Wang, Robins \& Pattison, 2006). It is a stand-alone software, it has both windows 731 .NET and Java versions. Because of the Java version, it can be considered as a cross-platform 732 application. Also, since it is not a library of some other languages and thanks to its user-friendly 733 environment, it is the most suitable choice for people with less computer programming 734 background. It is also a free software application and can easily be downloaded through its website 735 (http://www.melnet.org.au/pnet/).

736 Statnet (Handcock et al., 2003, 2008) is an R language package which can implement most state737 of-the-art ERGM methods and algorithms. It also has a variety of capabilities via other R libraries. 738 For example, some visualization options are available through libraries such as dynamic network 739 and rSoNIA. A wide range of network configurations has been implemented in this package. It has 740 an active community, and it seems that it is the de facto standard library for ERGMs. Thanks to its 741 open source and well-documented codebase, it can be used as a template for implementation of 742 new methods. However, because it is a programming language library and not standalone software, 743 it requires minimum knowledge of programming. (Goodreau et al., 2008) have presented a detailed 744 explanation for its installation and usage. There are also other extensions for the Statnet; for 745 example, (Caimo \& Friel, 2012) has incorporated the Bayesian ERGMs into the library.

\section{Conclusions}

747 This study offered an explanation of Exponential Random Graph Models aka ERGMs. We also 748 reviewed some state-of-the-art methods published after 2016. These articles either presented new 749 methods for fitting the ERGMs parameters or studied the possibility of using new network 750 configurations. Further, we did a comprehensive study of the research articles published by 751 scientists of multiple disciplines which have leveraged the applications of ERGMs in their fields 752 of interest. Multiple variation of the ERGM networks have been reviewed. We classified research 753 articles in seven plus one (other applications) categories. These included research works in medical 754 imaging, healthcare applications, economics and management, political science, missing data and 755 link prediction, scientific collaboration, Wireless Networks Modelling, and other applications. 756 Altogether, these studies provided valuable insight into the potential use of ERGMs in 757 interdisciplinary research. We also presented a brief description of the ERGMs tools and libraries 758 which can be used by scientists to conduct research like the research papers we presented. The 759 objective of this study was to develop an understanding of the ERGMs methods and applications 760 for those with limited knowledge about them. However, more in depth study for applications of 761 ERGMs in each special area of study is still needed. These domain specific studies can do further 762 analysis on the technical side of the ERGM modelling which was not a concern of our work. Some 763 potential future directions for future research are: 
764

765

766

767

768

769

770

771

772

773

774

775

776

777

778

779

780

781

782

783

784

785

786

787

788

789

790

791

792

793

794

795

796

797

798

799

800

801

802

803

804

805

- There are many good papers investigated the applications of Exponential Random Graphs from social science research community. However, there is a lack of interest among engineering community in these methods. Investigating the possibilities of using ERGMs in networked data in various field of engineering studies is a research path should be considered in the future. Some examples are studies on computer network topology, internet measurement which this statistical tool might be used for prediction of missing links or for the purpose of data augmentation etc.

- Multilayer networks are now widely studied in different disciplines e.g. transport and economical networks. Despite some good works using state of the art ERGMs methods for multi-layer networks there is still a lack of interest in using statistical tools like ERGMs for them comparing to other methods.

- The hype of deep learning (LeCun, Bengio \& Hinton, 2015) has made many new possibilists for combining them with traditional methods to achieve better estimation. To the best of our knowledge no work has been done to this date trying to leverage graph based deep learning methods alongside ERGMs.

- Despite the existence of comprehensive libraries for ERGMs like statnet, there is still no library for it written in Python. Since Python is the most used programming language in data science it is worthwhile to implement a powerful library for ERGMs modelling in Python. One possible way is to extend current widely used libraries like NetworkX (Hagberg, Swart \& S Chult, 2008) to include ERGMs in them.

- To the best of our knowledge there is no comprehensive research on comparison of ERGMs with newly presented generative graph models like NetGAN (Bojchevski et al., 2018).

\section{References}

Amati V, Lomi A, Mira A. 2018. Social network modeling. Annual Review of Statistics and Its Application 5:343-369.

An W, Ding Y. 2018. The Landscape of Causal Inference: Perspective from Citation Network Analysis. The American Statistician 72:265-277.

Anderson CJ, Wasserman S, Crouch B. 1999. A p* primer: Logit models for social networks. Social Networks 21:37-66.

Baggio S, Luisier V, Vladescu C. 2017. Relationships between social networks and mental health: An exponential random graph model approach among Romanian adolescents. Swiss Journal of Psychology 76:5.

Becker KR, Stojek MM, Clifton A, Miller JD. 2018. Disordered eating in college sorority women: A social network analysis of a subset of members from a single sorority chapter. Appetite.

Bianchi F, Casnici N, Squazzoni F. 2018. Solidarity as a byproduct of professional collaboration: Social support and trust in a coworking space. Social Networks 54:61-72.

Block P, Koskinen J, Hollway J, Steglich C, Stadtfeld C. 2018. Change we can believe in: comparing longitudinal network models on consistency, interpretability and predictive power. Social Networks 52:180-191.

Bojchevski A, Shchur O, Zügner D, Günnemann S. 2018. NetGAN: Generating Graphs via 
806

807

808

809

810

811

812

813

814

815

816

817

818

819

820

821

822

823

824

825

826

827

828

829

830

831

832

833

834

835

836

837

838

839

840

841

842

843

844

845

846

847

848

849

850

851

Random Walks. arXiv preprint arXiv: 1803.00816.

Bouranis L, Friel N, Maire F. 2017. Efficient Bayesian inference for exponential random graph models by correcting the pseudo-posterior distribution. Social Networks 50:98-108.

Bouranis L, Friel N, Maire F. 2018. Bayesian model selection for exponential random graph models via adjusted pseudolikelihoods. Journal of Computational and Graphical Statistics:1-13.

Byshkin M, Stivala A, Mira A, Krause R, Robins G, Lomi A. 2016. Auxiliary parameter MCMC for exponential random graph models. Journal of Statistical Physics 165:740-754.

Caimo A, Friel N. 2011. Bayesian inference for exponential random graph models. Social Networks 33:41-55.

Caimo A, Friel N. 2012. Bergm: Bayesian exponential random graphs in R. arXiv preprint arXiv:1201.2770.

Caimo A, Friel N. 2014. Bergm: Bayesian Exponential Random Graphs in R. Journal of Statistical Software 61.

Caimo A, Pallotti F, Lomi A. 2017. Bayesian exponential random graph modelling of interhospital patient referral networks. Statistics in medicine 36:2902-2920.

Campbell BW, Marrs FW, Böhmelt T, Fosdick BK, Cranmer SJ. 2019. Latent influence networks in global environmental politics. PloS one 14:e0213284.

Chatterjee S. 2016. An introduction to large deviations for random graphs. Bulletin of the American Mathematical Society 53:617-642.

Chatterjee S, Diaconis P. 2013. Estimating and understanding exponential random graph models. The Annals of Statistics 41:2428-2461.

Chen THY. 2018. Statistical Inference for Multilayer Networks in Political Science.

Coleman JS. 1958. Relational analysis: the study of social organizations with survey methods. Human organization 17:28-36.

Dellitalia J, Johnson MA, Vespa PM, Monti MM. 2018. Network analysis in disorders of consciousness: four problems and one proposed solution (Exponential Random Graph Models). Frontiers in Neurology 9:439.

Desmarais BA, Cranmer SJ. 2012. Statistical inference for valued-edge networks: The generalized exponential random graph model. PloS one 7:e30136.

Duxbury SW, Haynie DL. 2018. Building them up, breaking them down: Topology, vendor selection patterns, and a digital drug market's robustness to disruption. Social Networks 52:238-250.

Erdös P, Rényi A. 1959. On random graphs, I. Publicationes Mathematicae (Debrecen) 6:290297.

Fagan J, Eddens KS, Dolly J, Vanderford NL, Weiss H, Levens JS. 2018. Assessing Research Collaboration through Co-Authorship Network Analysis. Journal of Research Administration 49:76-99.

Fienberg SE. 2010. Introduction to papers on the modeling and analysis of network data. The Annals of Applied Statistics:1-4.

Frank O. 1981. A survey of statistical methods for graph analysis. Sociological methodology $12: 110-155$.

Frank O, Strauss D. 1986. Markov graphs. Journal of the american Statistical association 81:832842.

Gallemore C, Jespersen K. 2016. Transnational markets for sustainable development governance: the case of REDD+. World Development 86:79-94. 
852 Geyer CJ, Thompson EA. 1992. Constrained Monte Carlo maximum likelihood for dependent

853 data. Journal of the Royal Statistical Society. Series B (Methodological):657-699.

854

855

856

857

858

859

860

861

862

863

864

865

866

867

868

869

870

871

872

873

874

875

876

877

878

879

880

881

882

883

884

885

886

887

888

889

890

891

892

893

894

895

896

897

Goldenberg A, Zheng AX, Fienberg SE, Airoldi EM. 2010. A survey of statistical network models. Foundations and Trends ${ }^{\circledR}$ in Machine Learning 2:129-233.

Goodman LA. 1961. Snowball sampling. The annals of mathematical statistics:148-170.

Goodreau SM. 2007. Advances in exponential random graph ( $\left.\mathrm{p}^{*}\right)$ models applied to a large social network. Social Networks 29:231-248.

Goodreau SM, Handcock MS, Hunter DR, Butts CT, Morris M. 2008. A statnet Tutorial. Journal of statistical software 24:1.

Gupta B, Iyer SK, Manjunath D. 2008. Topological properties of the one dimensional exponential random geometric graph. Random Structures \& Algorithms 32:181-204.

Hagberg A, Swart P, S Chult D. 2008. Exploring network structure, dynamics, and function using NetworkX.

Hamilton M, Lubell M. 2018. Collaborative governance of climate change adaptation across spatial and institutional scales. Policy Studies Journal 46:222-247.

Handcock MS, Hunter DR, Butts CT, Goodreau SM, Morris M. 2003. Statnet: software tools for the statistical modeling of network data. Seattle, WA. URL: http://statnetproject. org.

Handcock MS, Hunter DR, Butts CT, Goodreau SM, Morris M. 2008. statnet: Software tools for the representation, visualization, analysis and simulation of network data. Journal of statistical software 24:1548.

Handcock MS, Jones JH. 2004. Likelihood-based inference for stochastic models of sexual network formation. Theoretical population biology 65:413-422.

Hanneke S, Fu W, Xing EP, others. 2010. Discrete temporal models of social networks. Electronic Journal of Statistics 4:585-605.

Hellmann JK, Hamilton IM. 2018. Intragroup social dynamics vary with the presence of neighbors in a cooperatively breeding fish. Current Zoology.

Holland PW, Leinhardt S. 1977. A dynamic model for social networks. Journal of Mathematical Sociology 5:5-20Holland, P. W., Leinhardt, S. (1977). A dyna.

Hunter DR. 2007. Curved exponential family models for social networks. Social Networks 29:216230.

Hunter DR, Handcock MS, Butts CT, Goodreau SM, Morris M. 2008. ergm: A package to fit, simulate and diagnose exponential-family models for networks. Journal of statistical software 24:nihpa54860.

Iyer SK, Manjunath D. 2006. Topological properties of random wireless networks. Sadhana 31:117-139.

Karamchandani N, Manjunath D, Yogeshwaran D, Iyer SK. 2006. Evolving random geometric graph models for mobile wireless networks. In: 2006 4th International Symposium on Modeling and Optimization in Mobile, Ad Hoc and Wireless Networks. 1-7.

Kenniche H, Ravelomananana V. 2010. Random geometric graphs as model of wireless sensor networks. In: 2010 The 2nd international conference on computer and automation engineering (ICCAE). 103-107.

Koskinen JH. 2004. Bayesian analysis of exponential random graphs-estimation of parameters and model selection. Research Report 2004: 2, Department of Statistics, Stockholm University.

Koskinen JH, Robins GL, Pattison PE. 2010. Analysing exponential random graph (p-star) models with missing data using Bayesian data augmentation. Statistical Methodology 7:366-384.

Peer) Comput. Sci. reviewing PDF | (CS-2019:04:37112:1:2:NEW 5 Mar 2020) 
898 Koskinen JH, Robins GL, Wang P, Pattison PE. 2013. Bayesian analysis for partially observed

899

900

901

902

903

904

905

906

907

908

909

910

911

912

913

914

915

916

917

918

919

920

921

922

923

924

925

926

927

928

929

930

931

932

933

934

935

936

937

938

939

940

941

942

943 network data, missing ties, attributes and actors. Social Networks 35:514-527.

Koskinen JH, Snijders TAB. 2007. Bayesian inference for dynamic social network data. Journal of statistical planning and inference 137:3930-3938.

Krause RW, Caimo A. 2019. Missing Data Augmentation for Bayesian Exponential Random Multi-Graph Models. In: International Workshop on Complex Networks. 63-72.

Krause RW, Huisman M, Steglich C, Sniiders TAB. 2018. Missing network data a comparison of different imputation methods. In: 2018 IEEE/ACM International Conference on Advances in Social Networks Analysis and Mining (ASONAM). 159-163.

Krivitsky PN. 2012. Exponential-family random graph models for valued networks. Electronic journal of statistics 6:1100.

Krivitsky PN, Handcock MS. 2014. A separable model for dynamic networks. Journal of the Royal Statistical Society: Series B (Statistical Methodology) 76:29-46.

de la Haye K, Embree J, Punkay M, Espelage DL, Tucker JS, Green Jr HD. 2017. Analytic strategies for longitudinal networks with missing data. Social Networks 50:17-25.

LeCun Y, Bengio Y, Hinton G. 2015. Deep learning. nature 521:436.

Leifeld P. 2018. Polarization in the social sciences: Assortative mixing in social science collaboration networks is resilient to interventions. Physica A: Statistical Mechanics and its Applications 507:510-523.

Leifeld P, Cranmer SJ, Desmarais BA. 2017. Temporal exponential random graph models with btergm: Estimation and bootstrap confidence intervals. Journal of statistical software.

Leifeld P, Fisher DR. 2017. Membership nominations in international scientific assessments. Nature Climate Change 7:730.

Li W, Bradshaw AE, Clary CB, Cranmer SJ. 2017. A three-degree horizon of peace in the military alliance network. Science advances 3:e1601895.

Lozano S, Gutiérrez E. 2018. A complex network analysis of global tourism flows. International Journal of Tourism Research.

Lusher D, Koskinen J, Robins G. 2012. Exponential random graph models for social networks: Theory, methods, and applications. Cambridge University Press.

Marrs FW, Campbell BW, Fosdick BK, Cranmer SJ, Böhmelt T. 2018. Inferring Influence Networks from Longitudinal Bipartite Relational Data. arXiv preprint arXiv:1809.03439.

Mellon J, Evans D. 2018. Forecasting Social Network Reaction to Disruption: Current Practices and New Directions.

Mellon J, Yoder J, Evans D. 2016. Undermining and strengthening social networks through network modification. Scientific reports 6:34613.

Metropolis N, Rosenbluth AW, Rosenbluth MN, Teller AH, Teller E. 1953. Equation of state calculations by fast computing machines. The journal of chemical physics 21:1087-1092.

Morris M, Handcock MS, Hunter DR. 2008. Specification of exponential-family random graph models: terms and computational aspects. Journal of statistical software 24:1548.

Müller TS, Grund TU, Koskinen JH. 2018. Residential Segregation and 'Ethnic Flight'vs. 'Ethnic Avoidance'in Sweden. European Sociological Review 34:268-285.

Osei A. 2018. Like father, like son? Power and influence across two Gnassingbé presidencies in Togo. Democratization:1-21.

Pan Z. 2018. Varieties Of Intergovernmental Organization Memberships And Structural Effects In The World Trade Network. Advances in Complex Systems:1850001.

Pattison P, Wasserman S. 1999. Logit models and logistic regressions for social networks: II.

Peer] Comput. Sci. reviewing PDF | (CS-2019:04:37112:1:2:NEW 5 Mar 2020) 
944

945

946

947

948

949

950

951

952

953

954

955

956

957

958

959

960

961

962

963

964

965

966

967

968

969

970

971

972

973

974

975

976

977

978

979

980

981

982

983

984

985

986

987

988

989

Multivariate relations. British Journal of Mathematical and Statistical Psychology 52:169193.

Penrose M, others. 2003. Random geometric graphs. Oxford university press.

van der Pol J. 2018. Introduction to Network Modeling Using Exponential Random Graph Models (ERGM): Theory and an Application Using R-Project. Computational Economics:1-31.

RA Fisher MA. 1922. On the mathematical foundations of theoretical statistics. Phil. Trans. R. Soc. Lond. A 222:309-368.

Raghavendra CS, Sivalingam KM, Znati T. 2006. Wireless sensor networks. Springer.

Robins G, Pattison P, Kalish Y, Lusher D. 2007a. An introduction to exponential random graph ( $\left.{ }^{*}\right)$ models for social networks. Social Networks 29:173-191.

Robins G, Pattison P, Wasserman S. 1999. Logit models and logistic regressions for social networks: III. Valued relations. Psychometrika 64:371-394.

Robins G, Snijders T, Wang P, Handcock M, Pattison P. 2007b. Recent developments in exponential random graph $\left(p^{*}\right)$ models for social networks. Social Networks 29:192-215.

Schmid CS, Desmarais BA. 2017. Exponential random graph models with big networks: Maximum pseudolikelihood estimation and the parametric bootstrap. In: Big Data (Big Data), 2017 IEEE International Conference on. IEEE, 116-121.

Scott TA, Thomas CW. 2017. Winners and losers in the ecology of games: Network position, connectivity, and the benefits of collaborative governance regimes. Journal of Public Administration Research and Theory 27:647-660.

Shang Y. 2009. Exponential random geometric graph process models for mobile wireless networks. In: 2009 International Conference on Cyber-Enabled Distributed Computing and Knowledge Discovery. 56-61.

Silk MJ, Croft DP, Delahay RJ, Hodgson DJ, Weber N, Boots M, McDonald RA. 2017. The application of statistical network models in disease research. Methods in Ecology and Evolution 8:1026-1041.

Silk MJ, Fisher DN. 2017. Understanding animal social structure: exponential random graph models in animal behaviour research. Animal Behaviour 132:137-146.

Silk MJ, Weber NL, Steward LC, Hodgson DJ, Boots M, Croft DP, Delahay RJ, McDonald RA. 2018. Contact networks structured by sex underpin sex-specific epidemiology of infection. Ecology letters 21:309-318.

Simpson SL, Hayasaka S, Laurienti PJ. 2011. Exponential random graph modeling for complex brain networks. PloS one 6:e20039.

Simpson SL, Moussa MN, Laurienti PJ. 2012. An exponential random graph modeling approach to creating group-based representative whole-brain connectivity networks. Neuroimage 60:1117-1126.

Sinke MRT, Dijkhuizen RM, Caimo A, Stam CJ, Otte WM. 2016. Bayesian exponential random graph modeling of whole-brain structural networks across lifespan. Neuroimage 135:79-91.

Smith JA. 2012. Macrostructure from microstructure: Generating whole systems from ego networks. Sociological methodology 42:155-205.

Snijders TAB. 1996. Stochastic actor-oriented models for network change. Journal of mathematical sociology 21:149-172.

Snijders TAB. 2002. Markov chain Monte Carlo estimation of exponential random graph models. Journal of Social Structure 3:1-40.

Snijders TAB, Pattison PE, Robins GL, Handcock MS. 2006. New specifications for exponential random graph models. Sociological methodology 36:99-153.

Peer] Comput. Sci. reviewing PDF | (CS-2019:04:37112:1:2:NEW 5 Mar 2020) 
990 Solo V, Poline J-B, Lindquist MA, Simpson SL, Bowman FD, Chung MK, Cassidy B. 2018. 991 Connectivity in fMRI: Blind Spots and Breakthroughs. IEEE transactions on medical $992 \quad$ imaging $37: 1537-1550$.

993 Song H, Cho J, Benefield GA. 2018. The Dynamics of Message Selection in Online Political 994 Discussion Forums: Self-Segregation or Diverse Exposure? Communication 995

Stauffer J, Pedraza Martinez A, Yan LL, Van Wassenhove LN. 2018. Asset Supply Networks in Humanitarian Operations: A Combined Empirical-Simulation Approach.

Stivala AD, Koskinen JH, Rolls DA, Wang P, Robins GL. 2016. Snowball sampling for estimating exponential random graph models for large networks. Social Networks 47:167-188.

Thiemichen S, Kauermann G. 2017. Stable exponential random graph models with non-parametric components for large dense networks. Social Networks 49:67-80.

Thurner PW, Schmid CS, Cranmer SJ, Kauermann G. 2019. Network Interdependencies and the Evolution of the International Arms Trade. Journal of Conflict Resolution 63:1736-1764.

Ulibarri N, Scott TA. 2016. Linking network structure to collaborative governance. Journal of Public Administration Research and Theory 27:163-181.

Wang P, Robins G, Pattison P. 2006. PNet: A program for the simulation and estimation of exponential random graph models. University of Melbourne.

Wang P, Robins G, Pattison P, Lazega E. 2013. Exponential random graph models for multilevel networks. Social Networks 35:96-115.

Williams NL, Hristov D. 2018. An examination of DMO network identity using Exponential Random Graph Models. Tourism Management 68:177-186.

Wilson JD, Denny MJ, Bhamidi S, Cranmer SJ, Desmarais BA. 2017. Stochastic weighted graphs: Flexible model specification and simulation. Social Networks 49:37-47.

Windzio M. 2018. The network of global migration 1990-2013: Using ERGMs to test theories of migration between countries. Social Networks 53:20-29.

You J, Ying R, Ren X, Hamilton W, Leskovec J. 2018. GraphRNN: Generating Realistic Graphs with Deep Auto-regressive Models. In: International Conference on Machine Learning. 5694-5703.

Zhang C, Bu Y, Ding Y, Xu J. 2018a. Understanding scientific collaboration: Homophily, transitivity, and preferential attachment. Journal of the Association for Information Science and Technology 69:72-86.

Zhang S, de la Haye K, Ji M, An R. 2018b. Applications of social network analysis to obesity: a systematic review. Obesity Reviews 19:976-988.

Zhang C, Zhai BY, Wu M. 2013. Link prediction of community in microblog based on exponential random graph model. In: Wireless Personal Multimedia Communications (WPMC), 2013 16th International Symposium on. IEEE, 1-6.

Zhang Y, Zhang H, Sun W, Pan C. 2014. Connectivity analysis for vehicular ad hoc network based on the exponential random geometric graphs. In: 2014 IEEE intelligent vehicles symposium proceedings. 993-998. 
Figure 1

Finite state automata of a MCMC procedure 


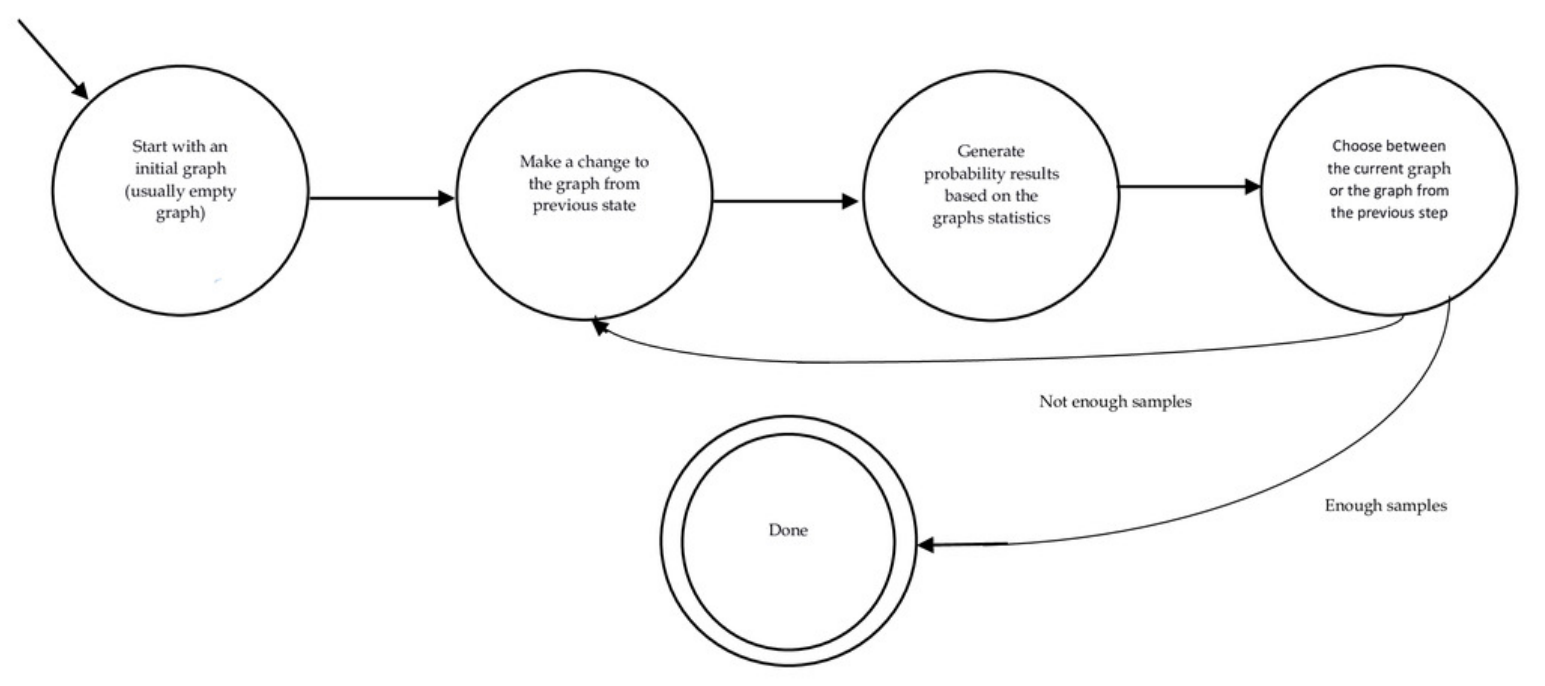


Figure 2

Procedure of fitting ERGMs variables 


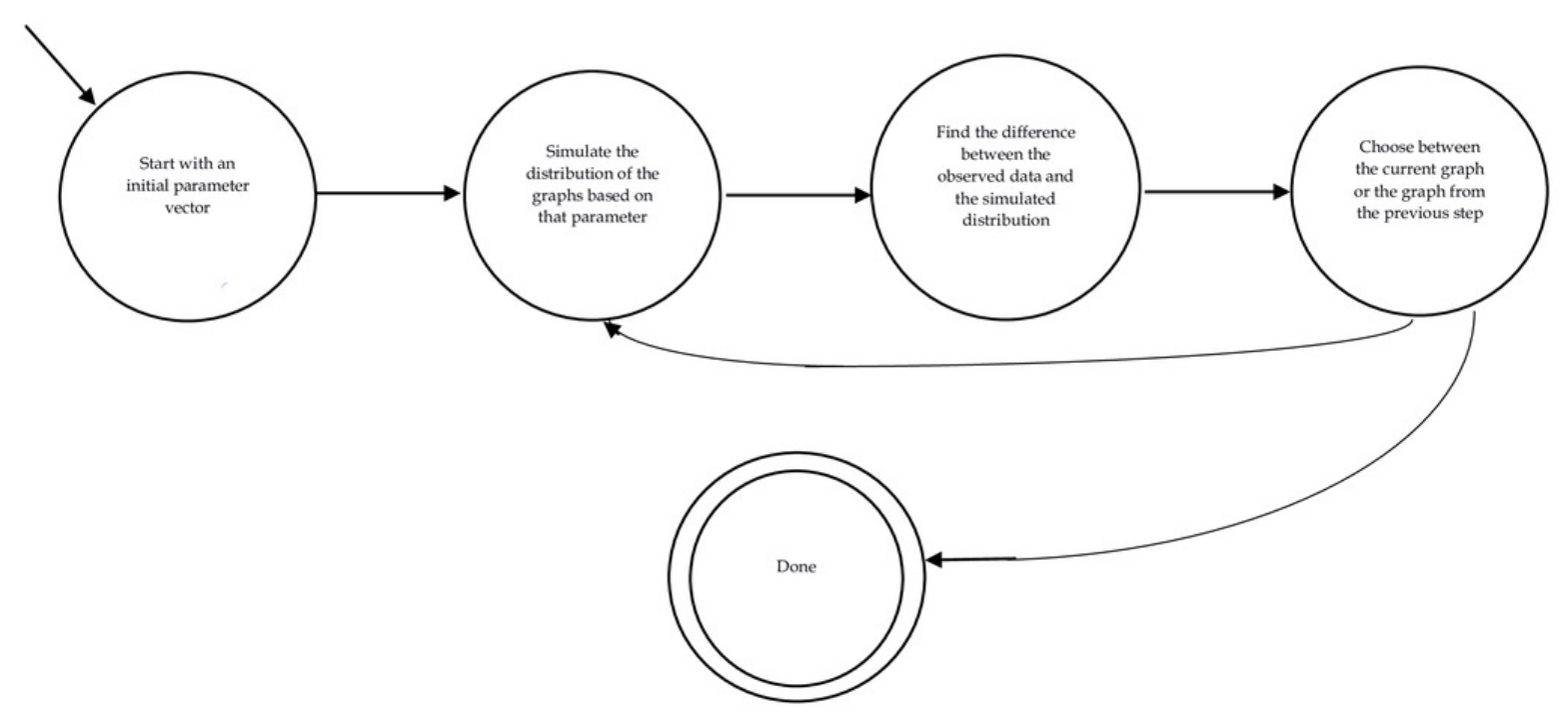




\section{Table 1 (on next page)}

Notations used throughout this work 
1

\begin{tabular}{cl}
\hline Notation & Meaning \\
$x$ & The set of all possible graphs with the same number of nodes. \\
$P$ & $\begin{array}{l}\text { The variable that indicates the presence of a particular graph from the } \\
\text { distribution. }\end{array}$ \\
$S$ & The probability distribution function of graphs. \\
$S$ & Some particular statistics of the network. \\
$C$ & The set of all count function of the network configurations. \\
$C$ & The count function of some particular statistics of the network. \\
$\theta$ & The set of all network statistics coefficients. \\
$N$ & Some particular statistics' coefficient of the network. \\
\end{tabular}

2 